\title{
A "Uniformity Principle" for Evacuation Route Allocation*
}

\author{
Richard L. Francis \\ Department of Industrial and Systems Engineering, University of Florida \\ Gainsville, FL 32611
}

April 1, 1981

\begin{abstract}
This paper establishes what might be called a "uniformity principle" for building evacuation problems. The principle may be stated as follows: given a building for which each occupant has reasonable access to every evacuation route, if the building is evacuated in minimum time, then the allocation of evacuees to routes is such that the route evacuation times are all the same. That is, there is a uniformity of route evacuation times. Also, analytical expressions for the minimum time to evacuate a building, and for the corresponding allocation of evacuees to routes, are obtained.
\end{abstract}

Key Words: building evacuation; mathematical optimization; network flow.

\section{Introduction}

The main purpose of this paper is to establish analytically what might be called a "uniformity principle" for building evacuation problems. The principle may be stated as follows: given a building for which the occupants have reasonable access to all the evacuation routes, if the building is evacuated in minimum time then the allocation of the people in the building to the various building evacuation routes is such that there is a uniformity of route evacuation times, that is, all route evacuation times are the same. This principle is easy to motivate. If the evacuation time for some route $j$ is greater than for all the other routes, then some people using route $j$ could be evacuated by other routes instead, thus reducing the time to evacuate route $j$ while not increasing the evacuation times for the other routes above the time to evacuate route $j$.

The uniformity principle appears to fall into the "folklore" category. People involved with building evacuation appear aware of the principle, and assume it is true, but only implicit references to the principle, such as the ones by Pauls and Jones [6], ${ }^{1}$ appear in the literature. As a consequence of the means by which we establish the principle, we obtain analytical expressions for the minimum time to evacuate a building, and for the number of people to be allocated to each evacuation route so as to achieve the minimum building evacuation time. We remark that even if the minimum building evacuation time is not achieved in an actual evacuation, it may still be of interest in the sense that it provides a benchmark, or standard of comparison, which gives some measure of how "good" an actual building evacuation time might be.

As concerns other related literature, for a graphical approach to the problems we shall consider, see Francis [3]. For various generalizations of the approach we shall consider, see Chalmet, Francis and Saunders [2], and Francis and Saunders [4]. For a general discussion of the evacuation literature, see Stahl and Archea [7].

The organization of the paper is as follows. We first give an analytical problem statement. We then give a solution procedure, followed by examples. Finally we consider and solve the more general problem where each route can have a capacity, i.e., can have an upper bound on the total number of people who can use the route. We motivate the correctness of the solution procedures we give, but omit proofs of correctness, as such proofs are relatively direct.

- This research was supported in part by the Center for Fire Research, National Bureau of Standards, Grant No. N879NAD0021, and by the 0perations Research Division, Center for Applied Mathematics, National Bureau of Standards.

${ }^{1}$ Figures in brackets indicate literature references at the end of this paper. 


\section{Analytical formulation}

So as to state the evacuation problem of interest analytically, suppose a building has $\mathrm{k}$ people to be evacuated, and there are $\mathrm{n}$ different evacuation routes. For $j=1, \ldots, n$ we assume we know that the time to evacuate $x_{j}$ people via route $j$, denoted by $t_{j}\left(x_{j}\right)$, is well structured, in the sense that it is a strictly increasing and continuous function with $t_{\mathcal{N}}(0)=0$. We call the function $t_{\mathcal{N}}()$ the route $j$ evacuation time function.

Since the building is not evacuated until all the routes are evacuated, the building evacuation time, say $z$, is the maximum, i.e., the longest, of the route evacuation times, that is,

$$
z=\max \left[t_{1}\left(x_{1}\right), \ldots, t_{n}\left(x_{n}\right)\right]
$$

Since we want to evacuate $k$ people, we require that

$$
x_{1}+\ldots+x_{n}=k
$$

that is, the total number of people evacuated via all the routes is equal to $k$. Since we cannot allow the number of people using any route to be negative, we also require

$$
x_{1} \geq 0, x_{2} \geq 0, \ldots, x_{n} \geq 0 \text {. }
$$

The evacuation problem can now be stated analytically: minimize (1) while satisfying (2) and (3). That is, find the (nonnegative) number of people to be evacuated via each route so as to minimize the time to evacuate the building.

Note it is assumed that routes do not "interact." For example, if routes 1 and 2 "cross" at some point, the time to evacuate route 1 would depend not only on $x_{1}$, the number of people using route 1 , but on $x_{2}$, the number of people using route 2 , as well; the model cannot handle such a situation. However, particularly for buildings for which the routes are essentially staircases, this assumption of no interaction appears reasonable. Further, in modeling a specific problem there may be more than one means of defining specific routes, in which case an appropriate definition might guarantee that in fact the routes do not interact.

It is also assumed that each of the $k$ persons can use any one of the $n$ routes; this assumption is implicit in the condition (2).

We emphasize the fact that the problem statement does not require $x_{j}$ to be an integer. Thus an implicit assumption is that solutions to the problem can be rounded to adjacent integers with an acceptable loss of accuracy. Particularly when the total number of people in the building is large in comparison to the number of routes, as is often the case, this assumption does not appear too restrictive. Further, if the integrality condition is imposed, then the uniformity principle may fail, although probably by only a little. For example, if a building contains 501 people, has two evacuation routes, and the two routes have the same route evacuation time functions, an optimum solution to the problem is to evacuate 250.5 people by each route, giving uniform evacuation times. In reality, the closest one would come to uniform evacuation times would be to evacuate 250 people via one route, and 251 via the other route, giving (slightly) different route evacuation times.

It is worth emphasizing that the route evacuation time functions need not actually be known in order for the uniformity principle to be true. It is only necessary to know that they are well structured. In particular, the route evacuation time functions need not be linear; nonlinearity makes the theory no more difficult. On the other hand, nonlinearity would certainly make the theory more difficult to implement computationally.

\section{Solution procedure}

We denote by $p_{j}(z)$ the number of people who can clear route $j$ in a time of $z$. Since $t_{j}\left(x_{j}\right)$ is the time to evacuate $x_{j}$ people via route $j$,

$$
z=t_{j}\left(p_{j}(z)\right)
$$


is the time to evacuate $p_{j}(z)$ people via route $j$, and so we obtain $p_{j}(z)$ by solving the equation (4) for $p_{j}(z)$. (Note we are just finding the inverse function of $t_{j}\left(\right.$ ) when we solve (4) for $p_{j}(\mathrm{z})$.) The expression (4) holds for z satisfying $0 \leq z \leq t_{j}(k)$. If $z>t_{j}(k)$ then the time $z$ is greater than that needed to evacuate all $k$ people via route $j$, and so in this case the number of people who can clear route $j$ in a time of $z$ is just $k$, i.e.,

$$
p_{j}(z)=k \text { for } t_{j}(k)<z \text {. }
$$

Thus we determine $p_{j}(z)$ either from (4) or from (5), depending upon whether $0 \leq z \leq t_{j}(k)$ or $t_{j}(k)<z$.

Note we can interpret $p_{j}(z)$ as the most people who can clear route $\mathrm{j}$ in a time of $z$, for if $x_{j}$ people clear route $j$, and $x_{j}>p_{j}(z)$, then, using (4) and the fact that $t_{j}()$ is a strictly increasing function, we have $t_{j}\left(x_{j}\right)>$ $t_{j}\left(p_{j}(z)\right)=z$, i.e., the time to clear more than $p_{j}(z)$ people exceeds $z$. Hence it follows, if we let

$$
P(z)=p_{1}(z)+\ldots+p_{n}(z) \text { for } 0 \leq z,
$$

that $P(z)$ is the most people who can exit the building in a time of $z$. Since there are $k$ people in the building, let us find the time $z^{*}$ for which

$$
k=P\left(z^{*}\right),
$$

in which case $k$ is the most people who can exit the building in a time of $z^{*}$. We can now conclude that in any time say $z^{\prime}$, with $z^{\prime}<z^{*}$, not all $k$ people can exit the building, and thus $z^{*}$ is the minimum time to evacuate all $k$ people.

Once we know $z^{*}$, we can allocate

$$
x_{j}^{*}=p_{j}\left(z^{*}\right) \text { people for each route } j
$$

and be assured that

$$
x_{1}^{*}+\ldots+x_{n}^{*}=p_{1}\left(z^{*}\right)+\ldots+p_{n}\left(z^{*}\right)=P\left(z^{*}\right)=k .
$$

Thus the allocation (8) evacuates all k people. Further, the time to evacuate $x_{j}^{*}$ people via route $j$ is given by

$$
t_{j}\left(x_{j}^{*}\right)=t_{j}\left(p_{j}\left(z^{*}\right)\right)=z^{*} \text { for each route } j .
$$

Thus every route clears at the same time, $z^{*}$. Therefore we obtain a uniformity principle, in the sense that the route evacuation times are uniform when the building is evacuated in minimum time.

At this point we summarize our procedure for allocating people to routes so as to evacuate the building in minimum time. First use (4) and (5) to determine each function $p_{j}()$. Then use (6) to find the function $\mathrm{P}(\mathrm{)}$. Given $k$, next solve (7) to find the minimum building evacuation time $z^{*}$. Then use (8) to determine an optimum allocation of people to routes, and conclude from (9) that all routes clear at the same time. We remark, if we suppose we draw every function $p_{j}()$, as well as the function $P()$, on a single graph, then we can envision all the steps of the procedure of this paragraph as being carried out using only this one graph.

We comment that the foregoing procedure is somewhat related to procedures given by Brown [1], but the applications he considers are quite different. Also, Brown does not consider the case where routes have capacities, which we treat in section 5 .

\section{Examples}

Let us consider some examples. As a simple initial example, suppose we can compute the time for $x_{j}$ people to clear route $j$ by dividing $x_{j}$ by a known (positive) flow rate $r_{j}$, so that $t_{j}\left(x_{j}\right)=x_{j} / r_{j}$ for every route $j$. Solving $z=p_{j}(z) / r_{j}$ for $p_{j}(z)$ gives $p_{j}(z)=r_{j} z$, so that $P(z)=r_{1} z+\ldots+r_{n} z=R z$, where $R=r_{1}+\ldots+r_{n}$. Thus solving $k=P\left(z^{*}\right)$ for $z^{*}$ gives $z^{*}=k / R$, which can be interpreted as the time to evacuate all $k$ people 
via a single-hypothetical-route of flow rate $R$. Similarly, $x_{j}^{*}=p_{j}\left(z^{*}\right)=r_{j} z^{*}=\left(r_{j} / R\right) k$ for every route $j$, and so the number of people allocated to each route $j$ is directly proportional to the flow rate of the route.

As a second example, consider a situation where a positive constant $c$ is known, as well as a positive constant $a_{j}$, a "route parameter," for each route $j$, and a strictly increasing and continuous function $t()$ with $t(0)=0$, so that $t_{j}\left(x_{j}\right)$ is computed as follows:

$$
t_{j}\left(x_{j}\right)=t\left(x_{j} /\left(c a_{j}\right)\right), 0 \leq x_{j} \leq k .
$$

By taking $t(y)=y, c=1$, and $a_{j}=r_{j}$ we get the previous example. By taking $c=1$ and $a_{j}=1$ for every route $j$ we get the situation where the time functions are the same for all routes. By taking $t(y)=y^{.73}, c=$ $(.206)^{1 / .73}$ and $a_{j}=w_{j}$ we get an empirically determined route time function of Pauls [5], where each route $j$ represents a stairwell and $w_{j}$ is the "effective width" in meters of stairwell $j$, obtained by subtracting 0.3 meters from the actual stairwell width. Thus this example represents a number of situations of interest. Let $p$ ( ) denote the function for which $z=t(p(z))$ and $y=p(t(y))$. By solving $z=t_{j}\left(p_{j}(z)\right)=t\left(p_{j}(z) /\left(c a_{j}\right)\right)$ for $p_{j}(z)$ we get $p(z)=p_{j}(z) /\left(c a_{j}\right)$, so that $p_{j}(z)=c a_{j} p(z)$. Letting $A=a_{1}+\ldots+a_{n}$ for convenience, we have $P(z)=p_{1}(z)+\ldots+p_{n}(z)=c A p(z)$. Thus solving $k=P\left(z^{*}\right)=c A p\left(z^{*}\right)$ for $z^{*}$ gives $z^{*}=t(k \wedge c A)$ ), which can be interpreted as the time for all $k$ people to clear a single-hypothetical-route having a route parameter $A$. The number of people allocated to each route $j$ is given by $x_{j}^{*}=p_{j}\left(z^{*}\right)=c a_{j} p\left(z^{*}\right)=c a_{j} p(t(k / c A))=$ $c a_{j}(k / c A)=\left(a_{j} / A\right) k$. Thus the number of people allocated to each route $j$ is directly proportional to its route parameter $a_{j}$. For the case where $t(y)=y, c=1, a_{j}=r_{j}$, and $A=R$ we get the same solution as in the previous example. By taking $c=1$ and every $a_{j}=1$, we have $A=n$, giving $x_{j}^{*}=k / n$ for every route $j$, so that when the time functions are the same for all $n$ routes the $k$ people are allocated evenly among the routes. For the case representing Pauls' time equation, letting $a_{j}=w_{j}$ and $A=W$ we get $x_{j}^{*}=\left(w_{j} / W\right) k$ for each route $j$, so that the number of people allocated to each route $j$ is directly proportional to the effective width of the route.

\section{Including roułe capacities}

We now consider briefly a capacitated problem, a generalization of the earlier (uncapacitated) problem defined by (1), (2), and (3). For each route $j$ we assume we know a capacity function $c_{j}()$, where $c_{j}(z)$ is an upper bound on the total number of people who can be evacuated via route $j$ in a time of $z$. For each $j$ we assume $c_{j}()$ is a continuous and nondecreasing function, with $c_{j}(0) \geq 0$. We obtain the capacitated problem by imposing the following capacity constraints upon the uncapacitated problem:

$$
x_{1} \leq c_{1}(z), \ldots, x_{n} \leq c_{n}(z), 0 \leq z
$$

Note that if we take each $c_{j}(z)=k$ in (10) that (10) becomes redundant, so that the capacitated problem includes the uncapacitated problem as a special case.

There are a number of reasons for considering capacity constraints. For example, a solution to the uncapacitated problem might allocate unrealistically large numbers of peoples to certain routes, which could be remedied by giving capacities to the routes in question. Alternatively, letting $c_{j}(z)$ be some constant $c_{j}$ might represent a situation where route $j$ becomes blocked after $c_{j}$ people exit the route. Further, the capacity functions permit the representation of situations where no extra people use the route in certain time intervals, e.g., if $c_{j}(30)=60=c_{j}(90)$ then the capacity of route $j$ is 60 at every point in time between $z=30$ and $z=90$, and if 60 people have cleared the route by the time $z=30$ then no extra people can clear the route until $z>90$ : such a situation might represent a temporary route blockage.

It turns out that the uniformity principle may fail for the capacitated problem, but the solution procedure remains much the same. For each route $j$, let $q_{j}(z)$ now denote the most people who can clear (capacitated) route $j$ in a time of $z$. Continuing to let $p_{j}()$ denote the function defined by (4) and (5), we have $q_{j}(z)=p_{j}(z)$ provided $p_{j}(z) \leq c_{j}(z)$, while $q_{j}(z)=c_{j}(z)$ if $c_{j}(z)<p_{j}(z)$, as the route capacity cannot be exceeded. Hence for each route $j$ we conclude

$$
q_{j}(z)=\text { minimum of } p_{j}(z) \text { and } c_{j}(z), 0 \leq z .
$$


With the definition (11) we can state the following procedure to solve the capacitated problem. First use (4) and (5) to determine each function $p_{j}()$, and then use (1l) to determine each function $q_{j}()$. Next construct the function $Q()$ defined by $Q(z)=q_{1}(z)+\ldots+q_{n}(z)$ for $0 \leq z$. If $k>Q(z)$ for all nonnegative $z$ then the capacity functions make it impossible to solve the problem as formulated, as all $k$ people can never be evacuated. Otherwise, given $k$, solve the equation

$$
k=Q(z)
$$

and take the minimum building evacuation time $z^{*}$ to be the smallest $z$ satisfying (12). (In case (12) has a unique solution take $\mathrm{z}^{*}$ to be the unique solution.) Then determine an optimum allocation of people to routes by letting $x_{j}^{*}=q_{j}\left(z^{*}\right)$ for each route $j$. As with the procedure for the uncapacitated problem, if we suppose we draw every function $q_{j}($ ), as well as the function $Q()$, on a single graph, then we can envision the steps of the procedure of this paragraph as being carried out using only this graph.

While the uniformity principle may fail, it can be shown that the principle still holds for routes that are not saturated. That is, every route $j$ for which $x_{j}^{*}<c_{j}\left(z^{*}\right)$ is cleared in a time of $z^{*}$.

Acknowledgement: The author would like to thank Dr. Alan J. Goldman for his many constructive suggestions.

\section{References}

[1] Brown, J. R. The knapsack sharing problem. Operations Research 27(2): 340-355; 1979 March-April.

[2] Chalmet, L. G.; Francis, R. L.; Saunders, P. B. Network models for building evacuation. Gainesville, FL: University of Florida, Department of Industrial and Systems Engineering; 1980 April; Research report 80-8. 41 p. (to appear in Management Science).

[3] Francis, R. L. A simple graphical procedure to estimate the minimum time to evacuate a building. Boston, MA: Society of Fire Prevention Engineers, 60 Batterymarch St.; 1979; SFPE technology report 79-5. 14 p.

[4] Francis, R. L.; Saunders, P. B. EVACNET: prototype network optimization models for building evacuation. Nat. Bur. Stand. (U.S.) NBSIR 79-1593, 1979 October. 107 p.

[5] Pauls, J. L. Management and movement of building occupants in emergencies. Ottawa, Canada: National Research Council of Canada, Division of Building Research; 1977 September. NRCC 16845, DDR Paper No. 788.

[6] Pauls, J. L.; Jones, B. K. Case studies of building evacuations (and) Building evacuation findings and recommendations, chapters in Behavior in Fires, D. Canter, ed. New York, NY: John Wiley \& Sons; 1978.

[7] Stahl, F; Archea, J. An assessment of the technical literature on emergency egress from buildings. Nat. Bur. Stand. (U.S.) NBSIR 77-1313, 1977 October. 57 p. 